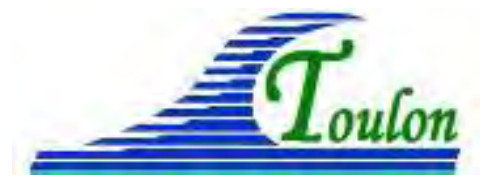
XIV ${ }^{\text {èmes }}$ Journées Nationales Génie Côtier - Génie Civil
Toulon, 29 juin au $1^{\text {er }}$ juillet 2016

DOI:10.5150/jngcgc.2016.024 @ Editions Paralia CFL

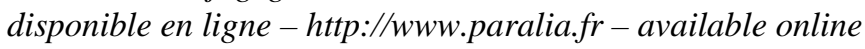

\title{
Modélisation morphodynamique à long terme pour la gestion sédimentaire du littoral portuaire dunkerquois
}

\author{
Camille JESTIN $^{1}$, Nicholas GRUNNET ${ }^{1}$, \\ Sten Esbjørn KRISTENSEN ${ }^{2}$, Nicolas FORAIN ${ }^{3}$
}

1. DHI France, Département Littoral et Offshore, 4 rue Edouard Nignon, CS 47202, 44372 Nantes Cedex 3, France.

caje@dhigroup.com;ngr@dhigroup.com

2. DHI Denmark, Coastal and Estuarine Dynamics, Agern Allé 5, 2970 Hørsholm, Denmark.skr@dhigroup.com

3. Grand Port Maritime de Dunkerque, Terre-plein Guillain, 59386 Dunkerque, France. nforain@portdedunkerque.fr

\section{Résumé :}

Le Grand Port Maritime de Dunkerque (GPMD) est situé dans un environnement morphodynamique complexe caractérisé notamment par de forts courants tidaux, un marnage important ainsi que par la présence de bancs sableux très mobiles.

De futurs travaux d'extension de l'avant-port Ouest vont rendre disponible jusqu'à $60000000 \mathrm{~m}^{3}$ de sédiment dans les années à venir. S'inscrivant dans l'optique d'une stratégie de gestion souple et durable de son littoral, le GPMD ne souhaite plus commercialiser les matériaux dont il dispose mais les valoriser en alimentant efficacement le littoral portuaire de Dunkerque, de façon à protéger durablement le littoral sans augmenter par ailleurs les besoins en dragage des chenaux de navigation.

Afin d'optimiser l'affectation de ce sable, DHI a mené une étude de modélisation numérique à l'aide du nouveau modèle MIKE21 FM Shoreline Morphology : un modèle d'évolution du trait de côte basé sur une approche hybride particulièrement adaptée à la modélisation à long terme, combinant une prise en compte 2D des processus gouvernant le transport sédimentaire à une réponse $1 \mathrm{D}$ en termes d'évolution du trait de côte.

L’impact et l'évolution de plusieurs scénarios de rechargement de plusieurs millions de mètres cubes de sédiment représentant des modifications massives de la morphologie du littoral portuaire dunkerquois ont été modélisés sur une période de dix ans afin de guider le GPMD dans l'élaboration d'un plan de gestion adapté à ses besoins et ses objectifs.

Mots-clés : Érosion, Evolution du trait de côte, Gestion sédimentaire, Modélisation, Morphodynamique, Rechargements, MIKE21, MIKE21 Shoreline Morphology FM.

\section{Introduction}

La politique de gestion du trait de côte a profondément évolué au cours des dernières années, passant d'une vision contrainte à une démarche plus environnementale, à des 


\section{Thème 2 - Dynamique sédimentaire}

interventions de défense contre la mer plus douces et à une meilleure connaissance des systèmes naturels. Une démarche basée sur des méthodes souples d'aménagement du littoral est désormais fortement encouragée et s'inscrit naturellement dans les objectifs du Grand Port Maritime de Dunkerque (GPMD), qui a mandaté DHI pour conduire des études hydro-sédimentaires dans le cadre de l'élaboration d'un plan de gestion du littoral entre les deux avant-ports de Dunkerque. Le GPMD fait face à différentes problématiques en termes de gestion sédimentaire : il connaît des besoins en dragages annuels importants afin de garantir des cotes d'exploitation suffisantes pour le trafic maritime, et est contraint de réaliser des rechargements de plage ponctuels pour pallier l'érosion chronique que subit l'essentiel de son linéaire côtier. Le plan de gestion devra répondre aux enjeux de protection du littoral dunkerquois dont l'érosion met en danger les infrastructures portuaires, en intégrant les opportunités offertes par la mobilisation de grandes quantités de sable issues d'une part des dragages d'entretien et d'autre part de futures opérations d'aménagement, qui devraient à terme fournir un volume de $60000000 \mathrm{~m}^{3}$ de sable valorisable.

\section{Présentation du site}

Le port de Dunkerque, troisième port de commerce français, est situé dans le Nord-Pasde-Calais, sur le littoral sableux de la plaine maritime flamande.

\subsection{Description morphodynamique}

Le domaine marin local se caractérise par une profondeur faible n'excédant pas $40 \mathrm{~m}$ et par une morphologie particulière, dominée par la présence de nombreux bancs sableux formant des hauts-fonds.

Le site d'étude (figure 1) en comporte quatre : les bancs du Braek et du Snouw, relativement stables à l'échelle de la dizaine d'années, sont séparés des bancs de Mardyck et de St-Pol par le chenal de navigation liant les avant-ports Ouest et Est. Les bancs de Mardyck et de St-Pol ont quant à eux montré au cours des dernières décennies une évolution très rapide, conséquence du réajustement de la morphologie du site suite à la construction de l'avant-port Ouest dans les années 70. Depuis 2000, le banc de Mardyck tend à se déplacer vers le Sud-Est au rythme de $70 \mathrm{~m} / \mathrm{an}$ tout en s'engraissant en sédiment, tandis que le banc de St-Pol disparaît peu à peu.

La granulométrie du site est caractérisée par un diamètre médian de l'ordre de 0,2 à $0,35 \mathrm{~mm}$.

Entre les avant-ports Ouest et Est s'étendent 14 km de linéaire côtier, caractérisé par deux types d'environnements distincts :

a) À l'Ouest : un massif dunaire (les dunes du Clipon) et une zone intertidale sableuse de faible pente à tendance érosive ;

b) À l'Est : une digue en enrobé bitumeux d'environ $6 \mathrm{~km}$ de long appelée digue du Braek à laquelle est accolée un large estran sableux en érosion chronique. 


\section{XIV èmes Journées Nationales Génie Côtier - Génie Civil \\ Toulon, 29 juin au $1^{\text {er }}$ juillet 2016}

L'érosion subie par le linéaire côtier depuis 2000 est estimée à -0,5 m/an. La dérive littorale, dirigée vers l'Est, est de l'ordre de 25000 à $35000 \mathrm{~m}^{3} / \mathrm{an}$, l'incertitude liée aux simulations de transport sédimentaire pouvant atteindre un facteur estimé de l'ordre de 2 à 4.

L'emprise de la présente étude comprend l'ensemble de la zone littorale entre les deux avant-ports, définie comme l'Unité de Gestion N4 (UG4) dans le Plan Littoral d'Action pour la Gestion de l'Erosion (P.L.A.G.E.) élaboré en 2003 par le Syndicat Mixte de la Côte d'Opale (SMCO \& ENR, 2003).

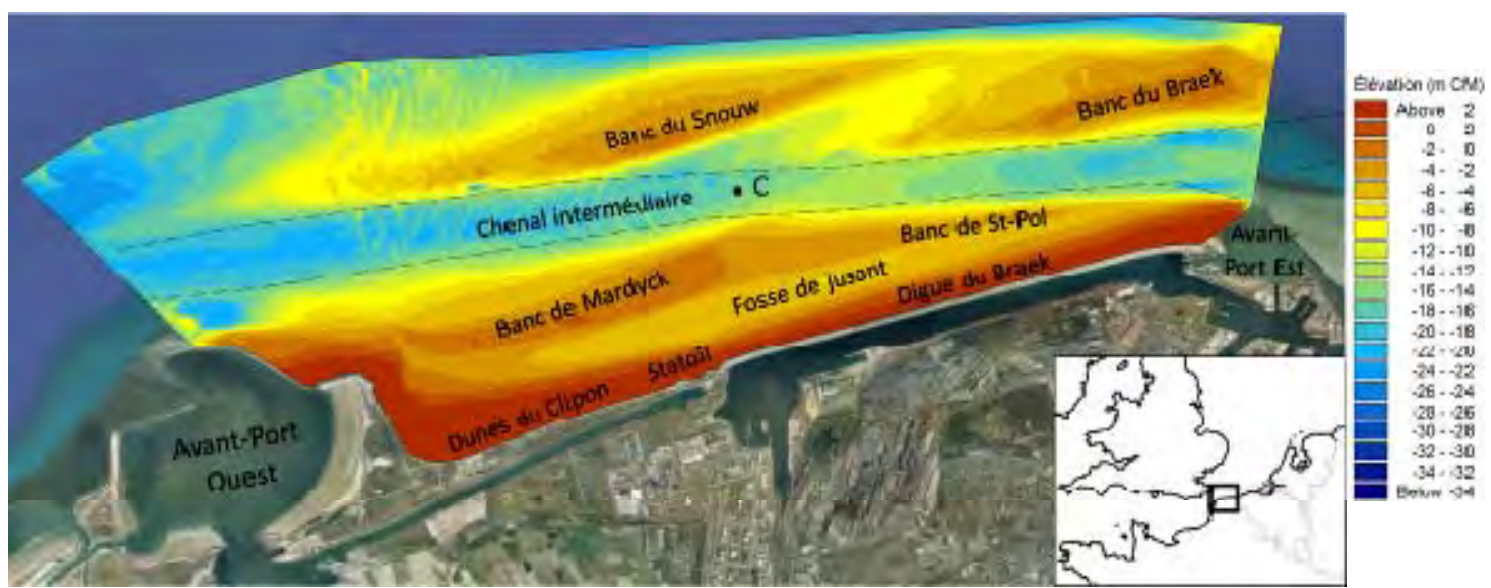

Figure 1. Localisation du site et bathymétrie de 2014. (Image satellite : Google Earth).

\subsection{Contexte hydrodynamique}

Le site d'étude est soumis à un régime de marée macrotidal de marnage moyen de l'ordre de 4,5 m. Les courants de marée sont particulièrement intenses au flot, vers le Nord-Est, où ils atteignent $1 \mathrm{~m} / \mathrm{s}$ (figure 2).

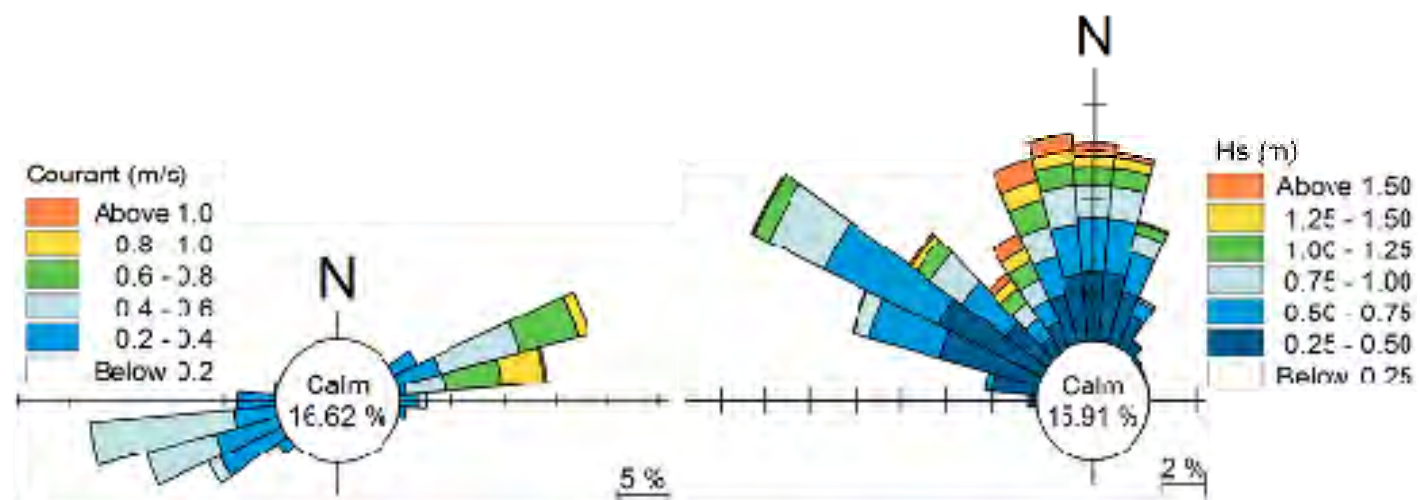

Figure 2. Roses des courants (à gauche) et des houles (à droite) modélisés au point $C$.

La dissymétrie des courants de marée entraîne un transport sédimentaire net dirigé vers l'Est. Le régime des houles au large connaît d'importantes variations saisonnières : d'avril à octobre les houles sont généralement calmes et en provenance de la Manche, 


\section{Thème 2 - Dynamique sédimentaire}

tandis que d'octobre à mars les conditions sont plus agitées avec des houles provenant principalement de la Mer du Nord. Les houles réfractent ensuite sur les bancs pour générer à la côte des houles dominantes de secteur Nord et Nord-Ouest (figure 2).

\section{Modélisation numérique}

\subsection{Présentation du modèle}

L'évolution et l'impact des scénarios de rechargement testés ont été modélisés sur 10 ans à l'aide du nouveau modèle morphodynamique côtier MIKE21 Shoreline Morphology FM de DHI, couplé dynamiquement avec les modules hydrodynamique, de houle spectrale et de transport sédimentaire non-cohésif de MIKE21. Ce nouveau modèle, basé sur une approche hybride, vise à combler le fossé existant entre les modèles de transport sédimentaire 2D détaillés, et les modèles d'évolution du trait de côte $1 \mathrm{D}$ adaptés aux simulations à long terme mais où les processus 2D sont inclus via une approche simplifiée. L'idée est d'utiliser un modèle bidimensionnel détaillé pour représenter les conditions hydrodynamiques et le transport sédimentaire, mais au sein du domaine du modèle côtier, découpé en sections le long du trait de côte (figure 3), des restrictions sont imposées sur les degrés de liberté de l'évolution morphologique en imposant l'orientation générale du trait de côte ainsi que la forme du ou des profil(s) caractéristique(s) du linéaire côtier. L'évolution morphologique dans le domaine du modèle côtier est par conséquent déterminée par l'intégration du transport sédimentaire sur le profil de plage, et est traduite pour chaque section en termes de translation du profil perpendiculairement au trait de côte (KAERGAARD \& FREDSØE, 2013, KRISTENSEN et al., 2010).

\subsection{Mise en œuvre du modèle}

Le modèle côtier a été appliqué sur la hauteur active avec une résolution de $100 \mathrm{~m}$ sur $10 \mathrm{~km}$ le long de l'estran (figure 3). En dehors de ce secteur, l'évolution de la morphologie est calculée par le modèle de transport sédimentaire non-cohésif de MIKE21 pour représenter la dynamique 2D des bancs et des fonds marins, et prendre ainsi en compte l'impact de leur évolution dans le temps sur l'évolution du trait de côte. Ainsi, la modélisation effectuée est véritablement 2D sur le bas-estran et les bancs subtidaux, et hybride sur l'estran.

Les données de forçage météo-marin sont issues de modèles courantologique et de houle spectrale régionaux de DHI, calés et validés sur des données mesurées. Afin d'accélérer les temps de calcul, le forçage hydrodynamique a été réalisé de façon synthétique. Ainsi, les niveaux d'eau ont été représentés par une marée morphologique de $10 \%$ supérieure au marnage moyen de la marée astronomique (GRUNNET et al., 2004). Les conditions de houles ont été schématisées en 51 états de mer représentatifs du climat de houle annuel (figure 4) : ils représentent 90\% du transport sédimentaire 


\section{XIV èmes Journées Nationales Génie Côtier - Génie Civil \\ Toulon, 29 juin au $1^{\text {er }}$ juillet 2016}

annuel généré par la houle. Chacun de ces états de mer est appliqué le temps d'un cycle de marée et associé à un facteur d'accélération de la morphodynamique dépendant de leur fréquence d'occurrence. Une analyse croisée de la houle et du vent a permis de définir le forçage éolien pour chacune des conditions de houles sélectionnées. Le modèle morphodynamique a été calé sur la période 2000-2013 puis validé sur la période 2013-2014 par des comparaisons avec des levés bathymétriques disponibles. La bonne reproduction de l'évolution récente des bancs subtidaux ainsi que des régimes d'érosion et d'accrétion le long de l'estran a ainsi pu être vérifiée.

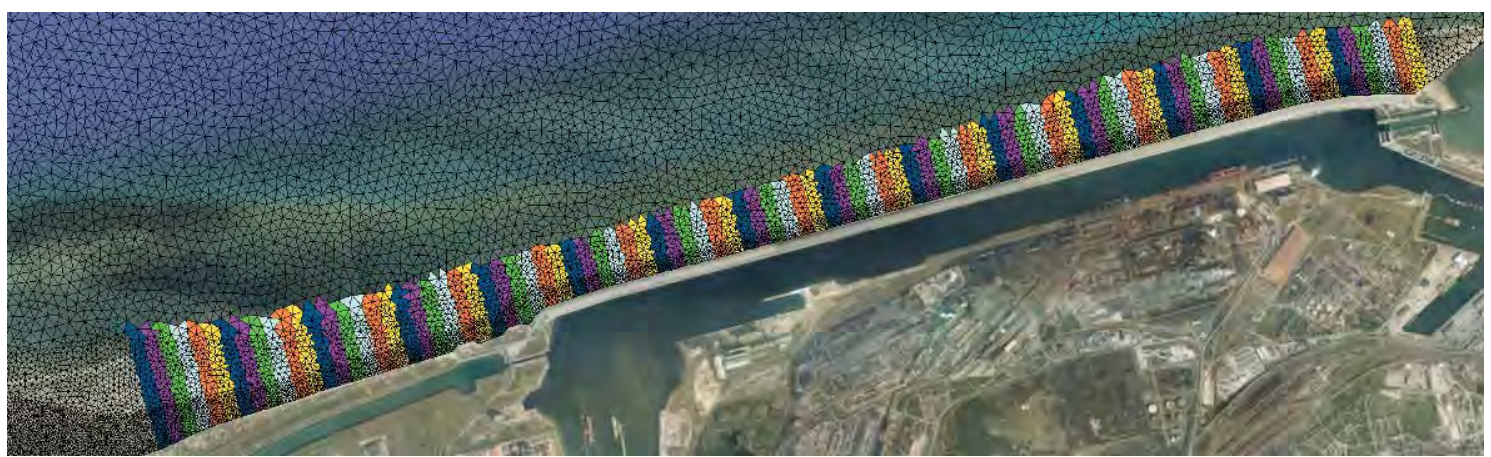

Figure 3. Détail du maillage (lignes noires) à la côte. Les sections du modèle côtier sont représentées en couleur. (Image satellite : Google Earth).
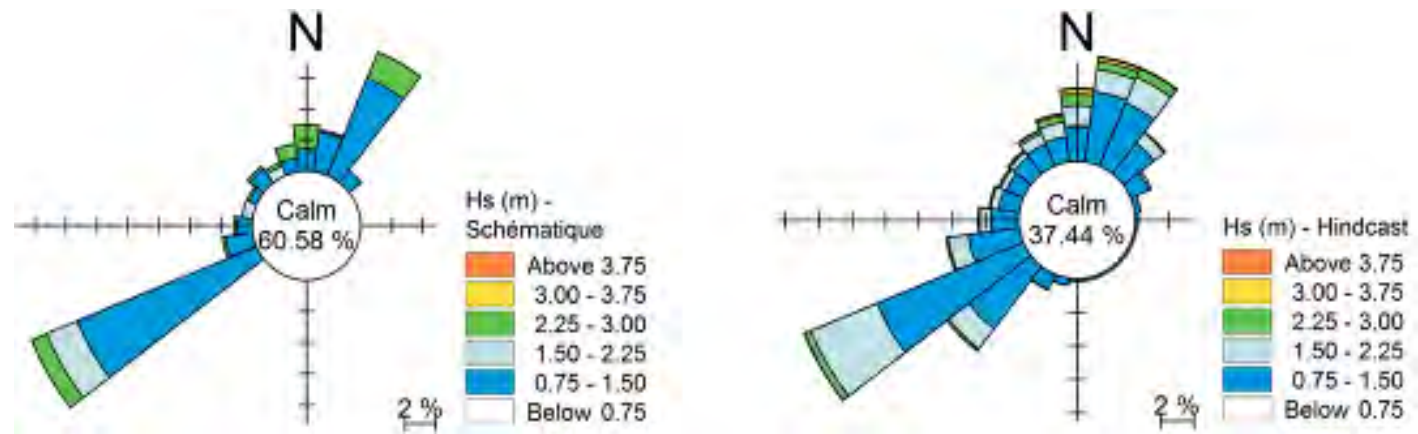

Figure 4. Roses des houles au large de Dunkerque schématisées (à gauche) et issues du modèle régional (à droite).

\section{Résultats de modélisation}

Après calage du modèle, l'impact sur l'évolution du trait de côte de différents scénarios de rechargements a été modélisé sur dix ans à partir des forçages synthétiques décrits précédemment. Du fait de l'importance des transports éoliens le long du littoral dunkerquois (TRESCA, 2013) qui génèrent des dépôts de sable conséquents dans les bassins portuaires et entraînent un besoin en dragages réguliers, des scénarios de rechargement de la zone subtidale ont été préférés à la possibilité de rechargements de plage massifs. Deux des scénarios les plus pertinents sont présentés ci-dessous. Ils sont 


\section{Thème 2 - Dynamique sédimentaire}

comparés à un scénario de référence correspondant à l'évolution sur dix ans sans intervention anthropique autre que les dragages d'entretien annuels.

\subsection{Formation d'un banc subtidal}

Un scénario envisagé consiste en la formation d'un banc subtidal de $4500000 \mathrm{~m}^{3}$ (volume nécessaire pour créer un banc suffisamment haut pour avoir un impact) dans l’alignement du flanc Sud-Est du banc de Mardyck (figure 4).

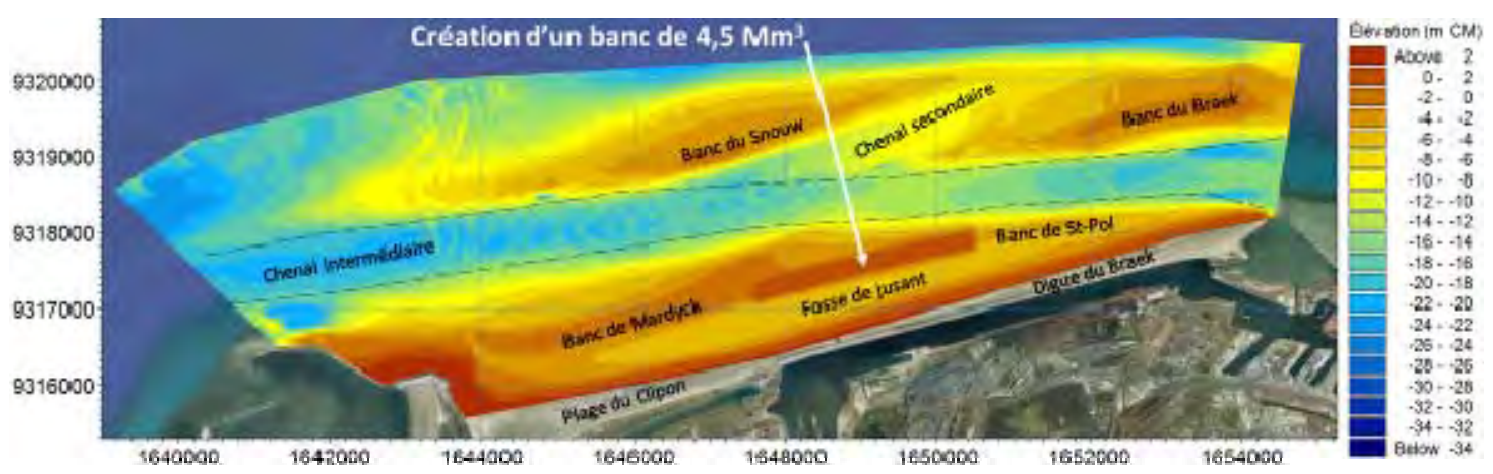

Figure 4. Scénario de formation d'un banc subtidal, bathymétrie de 2014 (à t=0).

Après dix ans de modélisation, le banc s'étend sur $3800 \mathrm{~m}$ de long, $300 \mathrm{~m}$ de large, et atteint la cote $+0,5 \mathrm{~m} \mathrm{CM}$ (figure 5, haut).

Ce scénario montre un impact localement très positif sur l'évolution du trait de côte (figure 5, bas). En agissant à la façon d'un brise-lames, il permet d'inverser le régime d'érosion chronique de l'estran en une avancée du trait de côte sur une distance de 2,5 $\mathrm{km}$ le long de la digue du Braek. Cependant, en ralentissant la dérive littorale à l'abri du banc, ce scénario génère une intensification sévère de l'érosion en aval (et dans une moindre mesure, à l'amont) de la zone impactée positivement. Ce scénario ne modifie pas le rythme d'érosion (toujours de l'ordre de 25000 à 35000 mªnn), mais réorganise les volumes de sable le long de l'estran.

\subsection{Formation d'un banc subtidal couplé à des rechargements de plage localisés}

Un second scénario basé sur la formation du banc subtidal précédemment décrit a été modélisé, mais couplé cette fois à deux rechargements de plage de $500000 \mathrm{~m}^{3}$ chacun afin de garantir une largeur de plage supplémentaire de $30 \mathrm{~m}$ sur les secteurs d'érosion mis en évidence par le scénario précédent. Les rechargements de plage opérés ont ainsi l'avantage d'apporter une protection immédiate à la digue, le long de laquelle l'érosion a atteint un seuil critique. La modélisation montre que la largeur de plage supplémentaire apportée par les rechargements permet de maintenir un bilan très positif (+16 m en moyenne) le long de l'estran, malgré l'érosion subie, toujours de l'ordre de 25000 à $35000 \mathrm{~m}^{3} /$ an (figure 6). 


\section{XIV ${ }^{\text {èmes }}$ Journées Nationales Génie Côtier - Génie Civil \\ Toulon, 29 juin au $1^{\text {er }}$ juillet 2016}

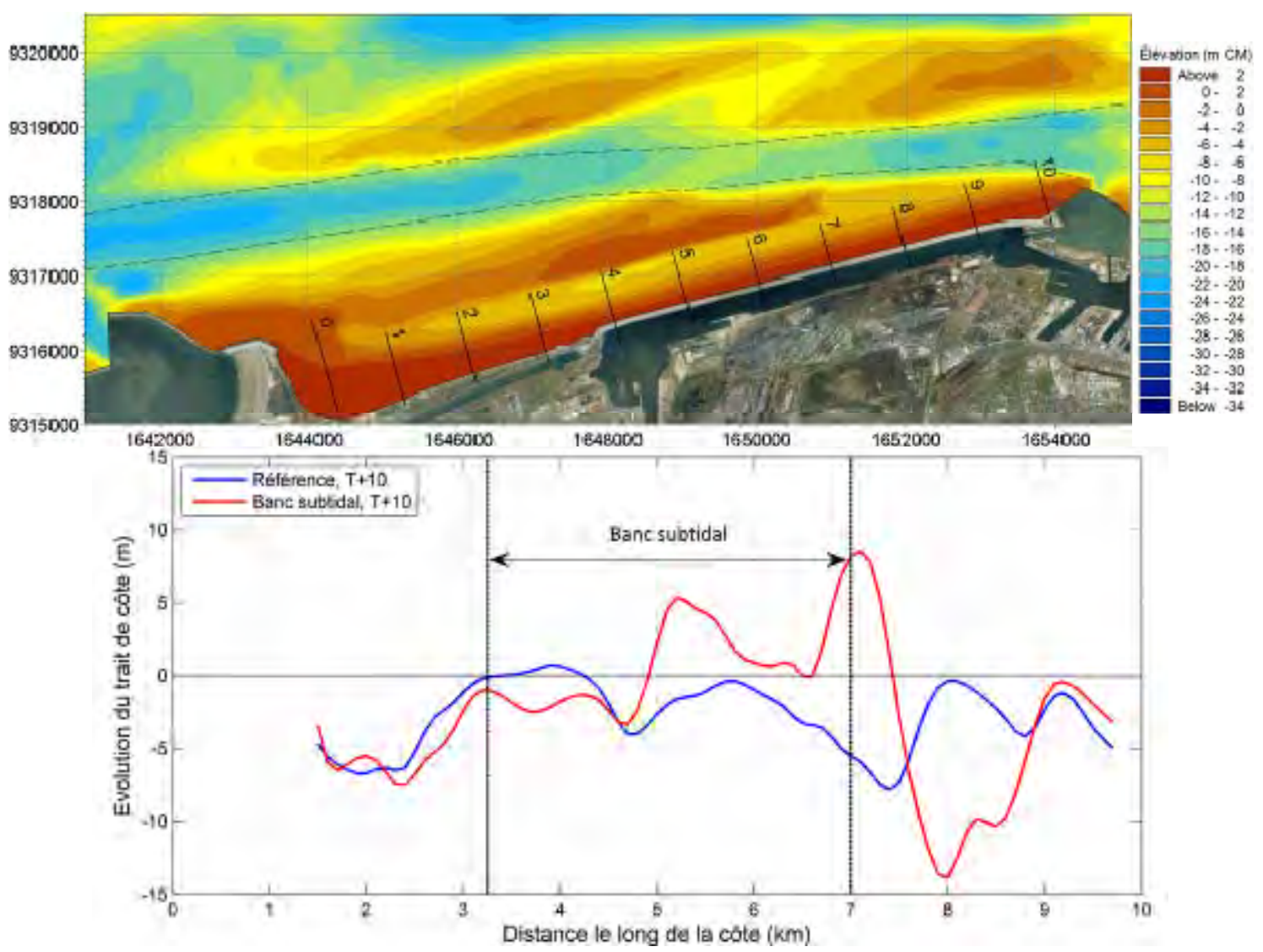

Figure 5. Bathymétrie (haut) et évolution du trait de côte (bas) après dix ans dans le cas du scénario de formation d'un banc subtidal.

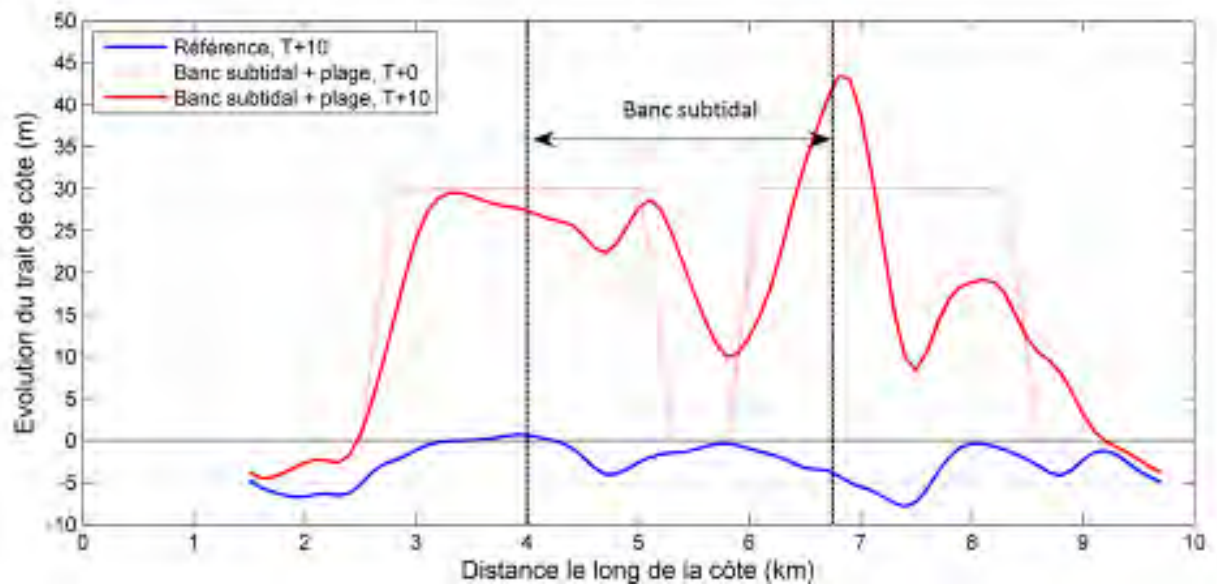

Figure 6. Evolution du trait de côte après dix ans dans le cas du scénario de formation d'un banc subtidal couplé à des rechargements de plage.

Par ailleurs, des bilans sédimentaires ont montré que ce scénario et le précédent sont sans effet significatif sur les besoins en dragages du chenal intermédiaire et des abords de l'avant-port Est. 


\section{Thème 2 - Dynamique sédimentaire}

\section{Conclusion}

Afin de remédier à la diminution progressive du stock sédimentaire du littoral portuaire dunkerquois, le GPMD souhaite valoriser les produits de dragage que des travaux d'aménagement futurs rendront disponibles en réalimentant son littoral.

Afin d'évaluer l'impact de rechargements massifs sur le trait de côte ainsi que sur les petits fonds du littoral portuaire dunkerquois, un modèle morphologique combinant la description 2D des champs de houle, de courant et de transport sédimentaire ainsi que l'évolution 1D du trait de côte a été construit: le modèle côtier MIKE21 Shoreline Morphology FM traduit ces conditions hydro-sédimentaires en termes d'avancée ou de recul du trait de côte, et l'ensemble permet d'avoir une vision globale de l'évolution de la morphologie du littoral dunkerquois tant au niveau des bancs subtidaux que de l'évolution du trait de côte sur les dix prochaines années pour plusieurs scénarios de rechargement. Certains scénarios ont montré un impact positif en termes de protection du littoral, en particulier la formation d'un banc subtidal. Couplé à des rechargements de plage localisés, ce scénario permet de maintenir après 10 ans de simulation une largeur de plage supplémentaire d'en moyenne $15 \mathrm{~m}$ par rapport à l'état actuel. Par ailleurs, ce scénario ne montre pas d'effets adverses en termes d'augmentation des besoins en dragages d'entretien des chenaux de navigation et limite les pertes sédimentaires dues au transport éolien.

Ces éléments, complétés par une étude de compatibilité granulométrique et de dispersion des sédiments fins en phase de travaux, permettront d'élaborer le plan de gestion du littoral dunkerquois selon les besoins du GPMD.

\section{Références bibliographiques}

GRUNNET N.M., WALSTRA D.J.R., RUESSINK B.G. (2004). Process-based modelling of a shoreface nourishment. Coastal Engineering, Vol. 51(7), pp 581607. http://dx.doi.org/10.1016/j.coastaleng.2004.07.016

KAERGAARD K., FREDSØE J. (2013). A numerical shoreline model for shorelines with large curvature. Coastal Engineering, Vol. 74, pp 19-32. http://dx.doi.org/10.1016/j.coastaleng.2012.11.011

KRISTENSEN S., GEIGAARD R., TAANING M., FREDSØE J., DRØNEN N., JENSEN J.H. (2010). Long term morphological modelling. Coastal Engineering Proceedings, No 32, sediment.64, 15 p.

SMCO, ENR -Syndicat Mixte de la Côte d'Opale, Espace Naturel Régional- (2003). Plan Littoral d'Actions pour la Gestion de l'Erosion sur le littoral de la Côte d'Opale. TRESCA A. (2013). Contrôle souple de la dynamique éolienne le long d'un littoral artificialisé et propositions de gestion : le cas de la façade maritime du Grand Port Maritime de Dunkerque. Thèse de doctorat, Université du Littoral Côte d'Opale. 